\section{Visión Electrónica \\ Más que un estado sólido}

https://revistas.udistrital.edu.co/index.php/visele

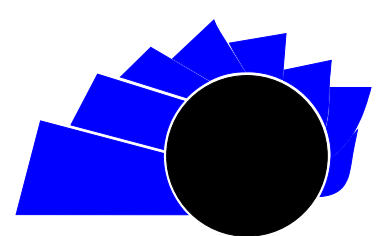

VISIÓN ELECTRÓNICA

VISIÓN INVESTIGADORA

\title{
Simulador de reparación de aneurismas cerebrales para entrenamiento médico
}

\author{
Simulator of Brain Aneurysm Repair for Medical Training
}

\begin{abstract}
Sergio Teodoro Vite. ${ }^{1}$, César Fabián Dominguez Velasco. ${ }^{2}$, Juan Bernardo Reséndiz Rodríguez. ${ }^{3}$, Aldo Hernández Valencia. ${ }^{4}$, Miguel Ángel Padilla Castañeda. ${ }^{5}$
\end{abstract}

\section{INFORMACIÓN DEL ARTÍCULO}

Historia del artículo:

Enviado: 13/09/2017

Recibido: 19/11/2017

Aceptado: 25/01/2018

Palabras clave:

Háptica

Interacción humano-computadora

Realidad virtual

Simulación en neurocirugía

Simulación por computadora

\section{Open access}

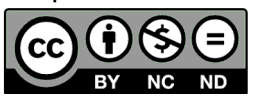

\section{Keywords:}

Haptics

Human-computer interaction

Virtual reality

Neurosurgery simulation

Computer simulation

\section{RESUMEN}

El desarrollo de sistemas de simulación de procedimientos quirúrgicos se ha convertido desde hace algunos años en un tema de investigación para diversas áreas que incluyen a la computación, la robótica y la medicina, pues suponen una alternativa novedosa para la adquisición de habilidades médicas, planeación, guía y control postoperatorio; al mismo tiempo que representan retos significativos en términos de su diseño, implementación y validación. En este trabajo se presentan las experiencias y metodologías aplicadas al desarrollo de simuladores computarizados para entrenamiento virtual en la Unidad de Investigación y Desarrollo Tecnológico (UIDT) en el Hospital General de México (HGM) "Dr. Eduardo Liceaga", de la Universidad Nacional Autónoma de México (UNAM). Como caso de estudio, se ejemplifica el desarrollo de un simulador para la reparación de aneurismas cerebrales, el cual ha involucrado la investigación en métodos de simulación computacional, visualización, sensaciones táctiles e interacción humano-computadora.

\footnotetext{
${ }^{1}$ Magíster en Ingeniería (Computación), Universidad Nacional Autónoma de México; doctor, Instituto de Investigaciones en Matemáticas Aplicadas y en Sistemas. Correo electrónico: sergioteovit@gmail.com, sergioteovit@comunidad.unam.mx

${ }^{2}$ Instituto de Investigaciones en Matemáticas Aplicadas y en Sistemas. Correo electrónico: isc.dominguezvc@gmail.com.

${ }^{3}$ Facultad de Ingeniería. Correo electrónico: jbernard.rr@gmail.com

${ }^{4}$ Unidad de Neurología y Neurocirugía, Hospital General de México “Dr. Eduardo Liceaga”. Correo electrónico:aldohernandez@me.com

${ }^{5}$ Doctor en Tecnologías de la Innovación y Robótica, Escuela Superior Sant Anna de Pisa; magíster en Ciencias (Computación), Universidad Nacional Autónoma de México. Investigador en la Unidad de Investigación y Desarrollo Tecnológico en el Hospital General de México "Dr. Eduardo Liceaga". Correo electrónico: miguel.padillacastaneda@gmail.com, miguel.padilla@ccadet.unam.mx
} 


\section{Introducción}

Neurosimulación [1] es el término empleado para describir el estudio, desarrollo y validación de sistemas capaces de recrear condiciones neurológicas y su correspondiente abordaje mediante técnicas quirúrgicas usando recursos tecnológicos. Este tipo de sistemas pueden clasificarse en tres tipos:

- HPS (siglas del inglés Human Patient Simulators): emplean modelos físicos o maniquíes construidos de materiales especiales al tacto, que pueden incluir complejos sistemas de control electrónico y por computadora como los descritos por [2].

- Simuladores computarizados: establecen escenarios idealizados en los cuales un usuario debe interactuar con un ambiente virtual y dispositivos automatizados para alcanzar una serie de metas, un ejemplo es Neurotouch [3], del National Research Council de Canadá.

- Simuladores híbridos: hacen uso de técnicas de realidad mezclada (realidad aumentada o virtualidad aumentada) que combinan maniquíes, modelos virtuales, dispositivos electrónicos y seguimiento con cámaras (tracking). Estos últimos sistemas se emplean generalmente para la planeación y guía en procedimientos quirúrgicos, como el sistema reportado por [4], donde se usa el concepto de neuronavegación para guiar a un usuario a través de venas y arterias cerebrales superpuestas virtualmente sobre un maniquí de nailon.

Desarrollar simuladores computarizados en neurocirugía implica adoptar retos importantes, debido a que se trata de recrear procedimientos que requieren de una alta precisión e involucran interacciones complejas [5]; por ejemplo, para simular el manejo de instrumental, se necesita de un dispositivo capaz de registrar los movimientos del cirujano y poder retroalimentar esas acciones con fuerzas que delimitan el espacio de trabajo. Para este último caso se recurre al uso de dispositivos de retroalimentación háptica comerciales o de "diseño a la medida", dependiendo de la aplicación médica.

Un procedimiento común y de alto riesgo en neurocirugía es la reparación de aneurismas cerebrales. Un aneurisma es una región débil de la pared de un vaso sanguíneo que provoca abultamientos, lo que tiene como consecuencia directa la alteración del flujo sanguíneo [6]. La complicación más grave de un aneurisma cerebral es que se rompa, pues conlleva a una acumulación de sangre en las regiones circundantes al cerebro o dentro de él.
En este sentido, los procedimientos estándares para la enseñanza de la reparación de aneurismas cerebrales hoy en día son: el clipaje y la reparación endovascular.

El clipaje de un aneurisma cerebral, desde el punto de vista de aprendizaje, es el resultado de una serie de pasos que un residente debe llevar a cabo para lograr un procedimiento exitoso. Uno de los sistemas más avanzados en el área de neurocirugía es Neurotouch [3], el cual se conforma por un conjunto de componentes tales como: un sistema de estereovisión, herramientas de manipulación háptica bimanuales y un software de simulación gráfico que conjunta la información visual, táctil y mecánica para ofrecer al usuario un ambiente de realidad virtual inmersivo en tiempo real.

\section{Desarrollo}

Actualmente, la UIDT del Centro de Ciencias Aplicadas y Desarrollo Tecnológico (CCADET) de la UNAM tiene un grupo de especialistas dedicado al desarrollo de simuladores computarizados, en los cuales se conjuntan conocimientos de ingeniería (eléctrica, electrónica, mecánica y computación) y medicina. Dentro de estos desarrollos, se encuentra el simulador computarizado para el entrenamiento médico en reparación de aneurismas cerebrales por clipaje. El sistema consiste en un software gráfico por computadora que funciona en tiempo real, en conjunto con dos dispositivos comerciales que proveen retroalimentación háptica, en el cual un aprendiz debe completar una serie de retos y acciones. La Figura 1 muestra el modelo conceptual, donde se resumen los niveles de destreza necesarios para que un residente en la especialidad de neurocirugía pueda efectuar un procedimiento de reparación de un aneurisma cerebral de manera exitosa.

\subsection{Modelo computacional}

Desde el punto de vista computacional, el sistema de simulación se compone de bloques funcionales que interactúan en tiempo real, como se muestra en la Figura 2. En general, es posible identificar cinco espacios sincronizados durante una simulación computarizada: hardware de despliegue gráfico (monitor y GPU, siglas del inglés Graphics Processing Unit) encargado de procesar todos los elementos visuales, modelos geométricos virtuales que definen la anatomía e instrumentos quirúrgicos (puntos, líneas, triángulos, tetraedros, etc.), modelos matemáticos que describen la biomecánica de los tejidos y objetos rígidos (métodos heurísticos, basados en la mecánica de los medios continuos e híbridos), modelos de colisión (árboles octan- 
Figura 1: Modelo conceptual de entrenamiento para residentes en neurocirugía para llevar a cabo un procedimiento de reparación de aneurismas cerebrales.
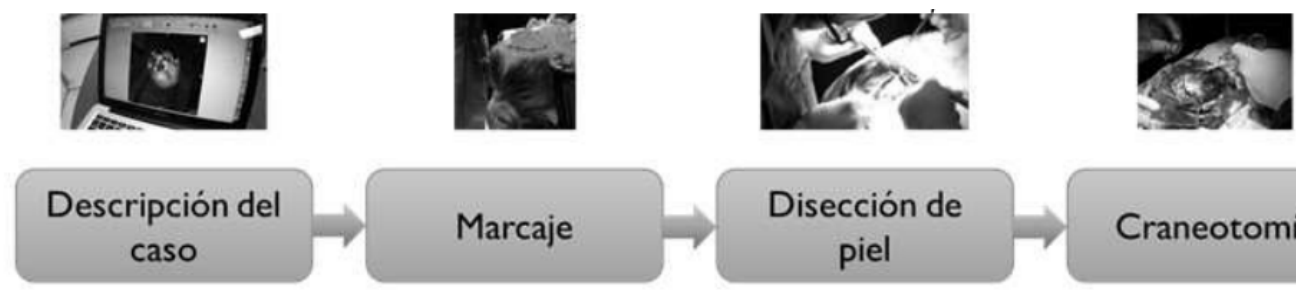

\section{Craneotomía}
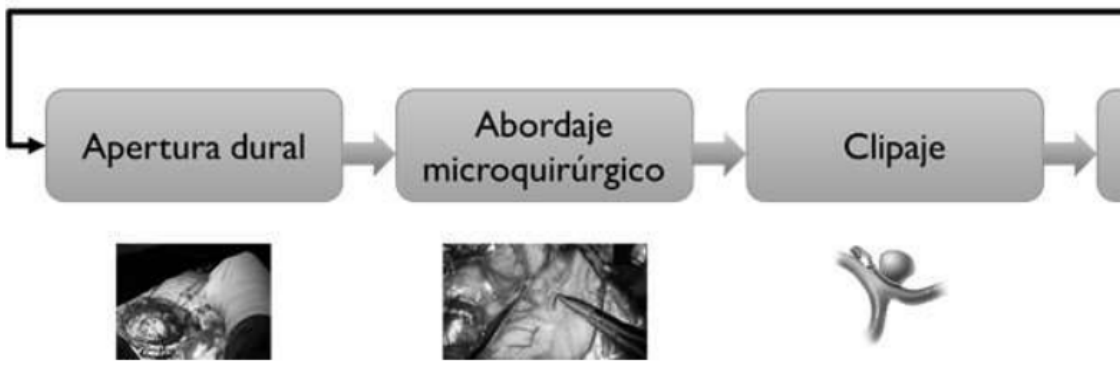

\section{Control post- operatorio}
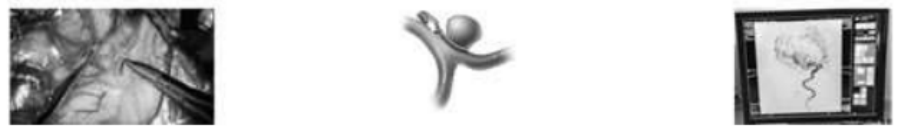

Fuente: elaboración propia.

tes, esferas y cajas de colisión, entre otras) y el modelo de despliegue táctil que proporciona al usuario la sensación de interactuar con tejido real, como por ejemplo, el cerebro, mediante dispositivos instrumentados.

Figura 2: Módulos principales para la simulación de objetos dinámicos y su interacción con el usuario dentro de un ambiente simulado por computadora.

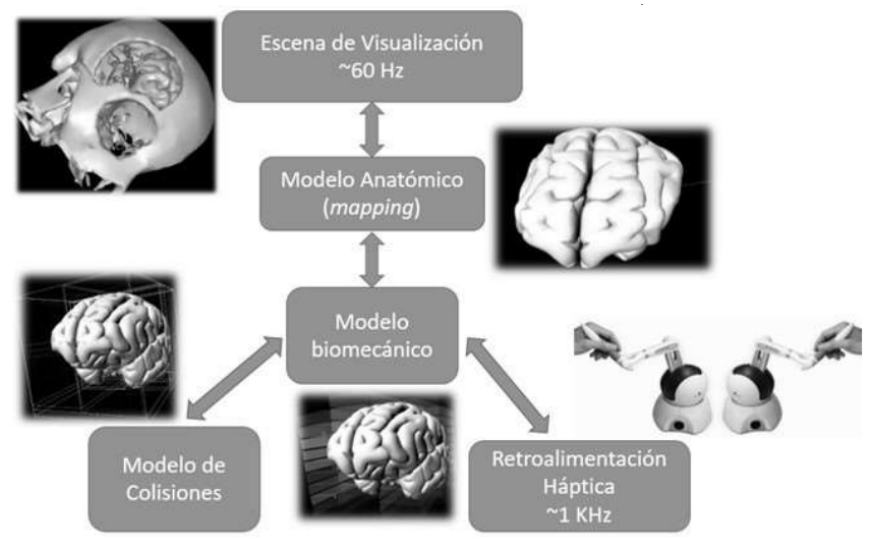

Fuente: elaboración propia.

\subsection{Modelado geométrico}

Los modelos geométricos definen un espacio de trabajo tridimensional delimitado por primitivas (puntos, líneas y superficies), las cuales describen objetos virtuales con formas simples como un cubo, o geometrías complejas como un modelo del cráneo, el cerebro o vasos sanguíneos. La Figuras 3 y la Figura 4 muestran ejemplos de reconstrucciones 3D de anatomías e instrumentos quirúrgicos, necesarios para representar visualmente objetos interactivos en los cuales un aprendiz puede identificar zonas de abordaje quirúrgico y seleccionar los instrumentos adecuados para ejecutar una acción dentro del sistema de simulación. Actualmente existen herramientas comerciales y de código abierto que auxilian en la obtención y manejo de estos modelos, tales como Osirix [7], Blender, MeshLab [8] y SimVascular [9].

Figura 3: Reconstrucción tridimensional de estructuras de piel y cráneo a partir de imágenes de angio-CT, usando OsiriX (los modelos son sometidos a una etapa de filtrado manual y reducción de triángulos con software como Blender y MeshLab) [7, 8].
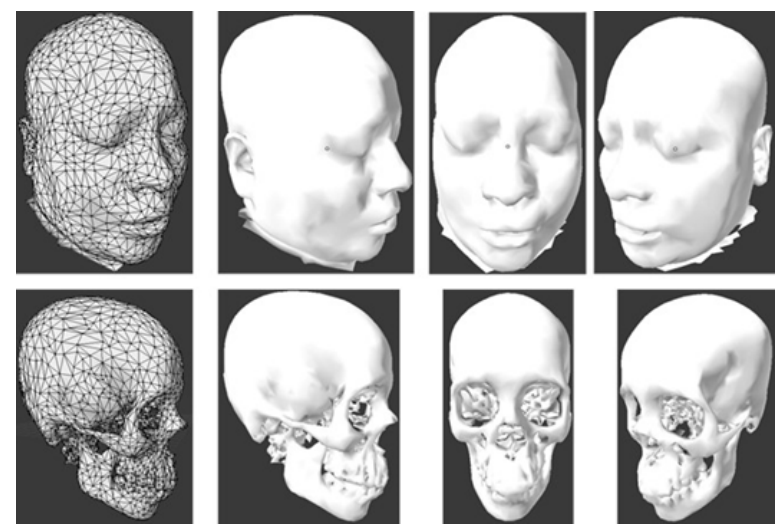
Figura 4: Modelos virtuales de clips en posición abierta y cerrada (izquierda). A la derecha, instrumentos de aplicación de clips y microtijeras modeladas en Blender a escala 1:1 con respecto a las hojas de especificaciones del fabricante.
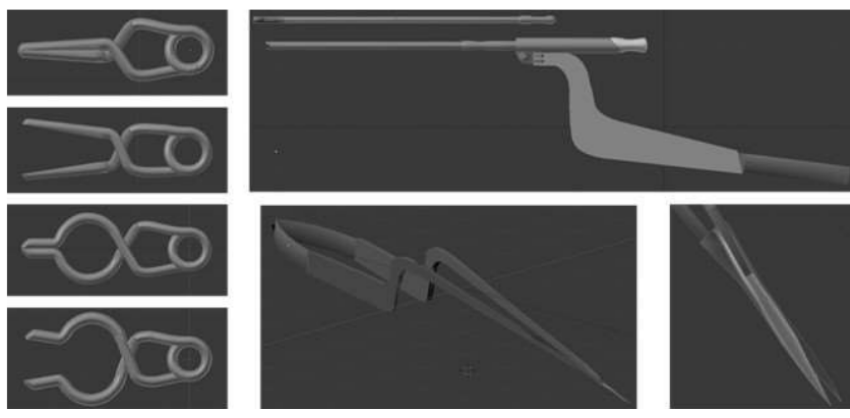

Fuente: elaboración propia.

\subsection{Modelado biomecánico}

El modelo biomecánico, tiene por objetivo establecer una relación interacción, simulación y realismo, en la cual, un modelo geométrico pueda ser llevado de un espacio estático a un espacio dinámico, donde el tiempo y las fuerzas involucradas establecen un modelo físico y matemático. Así, es posible simular computacionalmente tres tipos de objetos: rígidos, cuerpos deformables y partículas. En neurocirugía es importante representar estos tres tipos de objetos dependiendo de la estructura a abordar; así, por ejemplo, el cráneo puede considerarse como un objeto rígido, el cerebro como un cuerpo deformable y el flujo sanguíneo como un conjunto de partículas que fluyen a través de vasos sanguíneos y arterias.

En la literatura existen muchos trabajos acerca de los métodos de simulación para cada uno de los tipos de objetos, además, se han desarrollado bibliotecas comerciales y abiertas que implementan diversos métodos de simulación a nivel de hardware, por medio de GPU y unidades de procesamiento físico (PPU, por sus siglas en inglés), y software. Entre estas bibliotecas destacan: SOFA (siglas del inglés Simulation Open Framework Architecture [10]) del Institut National de Recherche en Informatique et en Automatique (INRIA) y Flex [11, 12] de la empresa NVIDIA.

SOFA es un motor de simulación y un entorno de trabajo que implementa el concepto de representación multimodelo basada en grafos de escena. Un grafo de escena está compuesto por un número arbitrario de objetos que interactúan bajo determinado conjunto de restricciones espaciales y ecuaciones, las cuales describen el comportamiento biomecánico de los objetos. La gran ventaja de emplear esquemas multimodelo, es que se puede manejar cada espacio de trabajo de manera independiente para después sincronizarlos mediante una operación llamada de "mapeo"; así, durante una simulación se definen tres espacios principales: un espacio geométrico de visualización, un espacio de colisiones (contactos entre objetos) y un espacio mecánico sobre el cual opera el modelo biomecánico como se muestra en la Figura 5.

Figura 5: Representación multimodelo de un objeto deformable en SOFA: (a) modelo visual o geométrico, (b) espacio biomecánico definido como un conjunto de hexaedros que envuelven el modelo geométrico (FEM, siglas del inglés Finite Element Method/FFD, siglas del inglés Free Form Deformation), (c) espacio de colisiones que emplea el algoritmo de árboles octantes, (d) mapeo entre espacios [10].
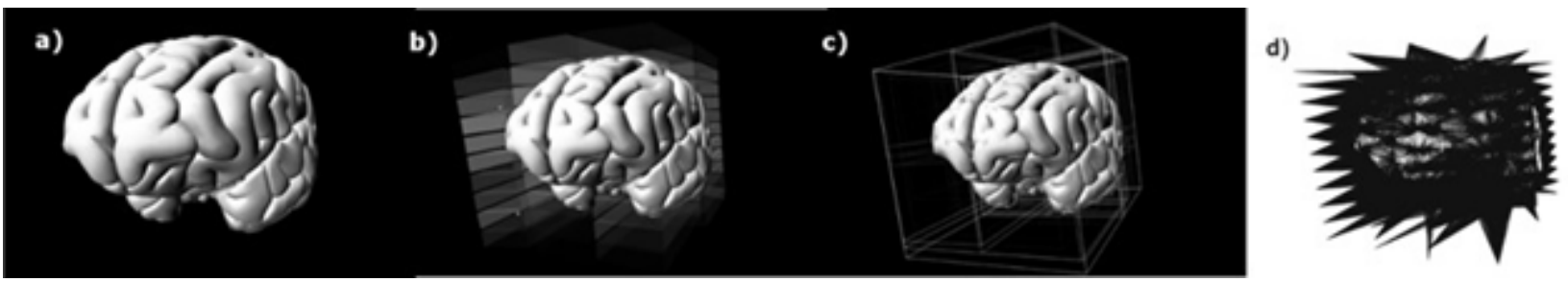

Un modelo matemático dinámico, ampliamente empleado en la literatura para simular la deformación de tejidos $[13,14]$ se basa en la mecánica de los medios continuos y de manera generalizada se rige por la ecuación del movimiento en su formulación lagrangiana.

$$
M \underline{\ddot{u}}+D \underline{\dot{u}}+K \underline{u}=\underline{f}
$$

Donde $M$ es la matriz de masa, $\ddot{u}$ es el vector de aceleración de la deformación, $D$ es el amortiguamiento, $\underline{\dot{u}}$ la velocidad de la deformación, $K$ la matriz de rigidez (definida en términos del módulo de Young y el radio de Poisson), $\underline{u}$ es el vector de deformación y $f$ es la suma de fuerzas externas que actúan sobre el modelo geométrico.

Para resolver la ecuación (1), se emplean diversos 
métodos de simulación, como el de FEM, el cual opera sobre mallas de tetraedros, y el método FFD que opera generalmente (más no exclusivamente) sobre mallas de hexaedros. La experimentación con estos métodos ha mostrado que son viables al usarse en sistemas de simulación para entrenamiento médico. Otros métodos, como el FEM-Co-rotacional [15,16] y técnicas de descomposición de dominio [14], también parecen ofrecer ventajas sustanciales en términos de realismo e interacción en tiempo real.

\subsection{Ambiente virtual}

Cada modelo geométrico y su correspondiente modelo biomecánico, convergen en un sistema de realidad virtual para integrar otro componente muy importante: la retroalimentación háptica. Los dispositivos hápticos, como el Omni Phantom de la empresa Geomagic, proveen un mecanismo de registro sobre cada una de las posiciones del instrumento simulado físico, manipulado por el usuario, que estima las fuerzas aplicadas en el modelo virtual (fuerzas externas). Estas fuerzas son resueltas en el modelo biomecánico, el cual devuelve como resultado un vector de fuerza correspondiente a la respuesta táctil. El vector de fuerza es enviado al dispositivo, el cual, por medio de vibro-motores, genera una oposición al movimiento.

Por otra parte, el estímulo visual ofrece al usuario una ventana a un supuesto escenario real, que en neurocirugía implica la representación realista de estructuras como piel, cráneo, cerebro, vascularidades e instrumental quirúrgico. La Figuras 6 y la Figura 7 muestran algunas pruebas experimentales donde se ejemplifica el despliegue gráfico con una acción simulada de colocación de un clip sobre el cuello de un aneurisma cerebral.

Figura 6: Prueba de clipaje. A la izquierda, vista externa del área de abordaje con aperturas de piel, cráneo y cerebro expuestas (modelos geométricos idealizados). Al centro, visualización de vascularidades con efectos de iluminación. A la derecha, vista del clip posicionado en el cuello del aneurisma.
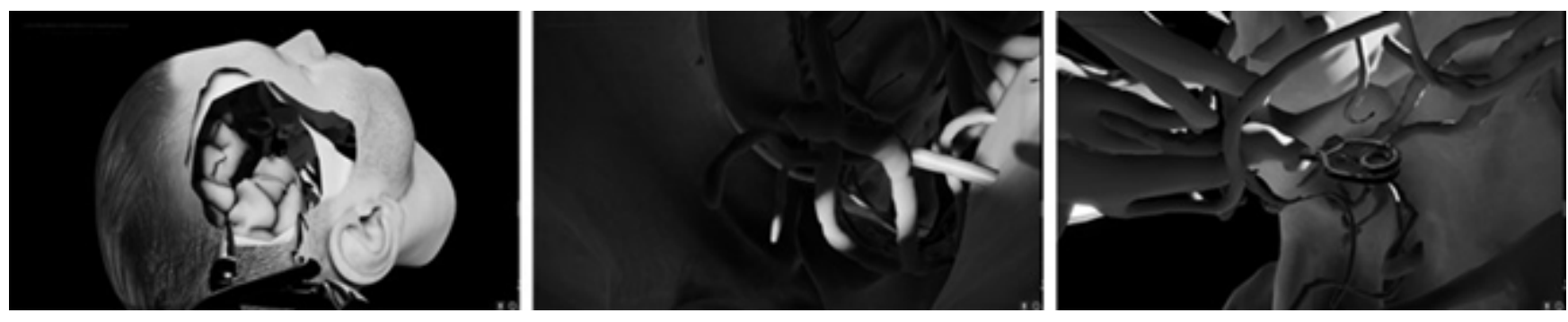

Fuente: elaboración propia.

Figura 7: Simulación de colocación de clip en el ambiente virtual.

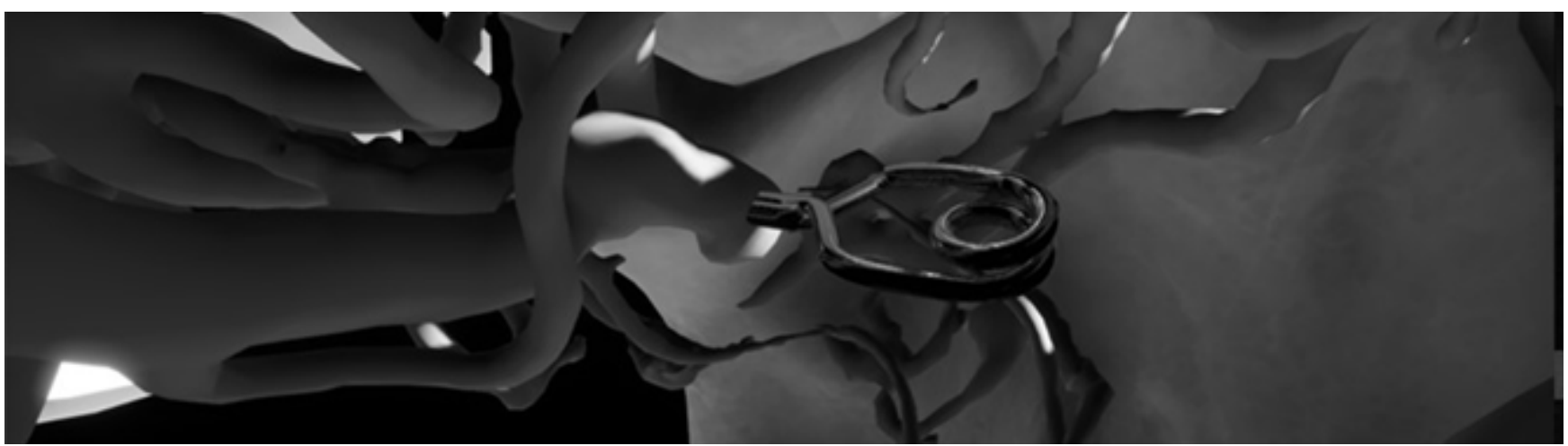

Fuente: elaboración propia. 


\subsection{Entrenamiento virtual}

El desarrollo del sistema de realidad virtual para reparación de aneurismas, en su estado actual, considera la validación con aprendices en la especialidad en neurocirugía. Las pruebas van enfocadas a la definición de métricas que permitan definir parámetros de aprendizaje a partir de comparaciones entre cirujanos expertos y novatos.

Un primer experimento se llevó a cabo con un sistema de abordaje quirúrgico a través de una apertura craneal. Este prototipo experimental aplica un análisis estadístico sobre la trayectoria empleada por el cirujano para realizar un procedimiento de clipaje. En busca de monitorear la validez de la trayectoria, se colocan puntos de control sobre un modelo gráfico, los cuales deben ser alcanzados a fin de determinar si la tarea fue realizada con éxito; posteriormente, se realiza una evaluación de la trayectoria que siguen las herramientas virtuales y se generan tres posibles aperturas. El cirujano vuelve a repetir la tarea usando cada una de las aperturas propuestas, con lo que se puede hacer una evaluación de la mejor apertura con base en su velocidad.

\section{Conclusiones}

En la versión actual del prototipo, la integración de los métodos computacionales de la biblioteca SOFA [10] para resolver el comportamiento biomecánico de las anatomías virtuales (principalmente tejido cerebral) y los dispositivos de retroalimentación háptica, aumentaron el grado de realismo percibido por el usuario con respecto a versiones anteriores, en las cuales no se consideraba la deformación debida a la interacción con el instrumental virtual. De las pruebas realizadas, el método basado en elementos finitos FFD, parece ofrecer una ventaja significativa para la simulación en tiempo real con respecto a otros métodos; sin embargo, se ha visto que ello depende del tipo de interacción involucrado en el proceso de simulación. Se espera con este nuevo prototipo llevar a cabo nuevos estudios de validación con residentes en el área de neurocirugía y así evaluar la utilidad de este tipo de sistemas en la enseñanza de la medicina.

\section{Referencias}

[1] A. Papangelou y W. Ziai, "The birth of neuro-simulation", Neurocrit Care, vol. 13, $\mathrm{n}^{\circ}$. 2, pp. 167-168, 2010. https://doi.org/10.1007/ s12028-010-9440-4

[2] M. J. Musacchio, A. P. Smith, C. A. McNeal, L. Munoz, D. M. Rothenberg, K. A. Von Roenn y R.
W. Byrne, "Neuro-critical care skills training using a human patient simulator", Neurocrit Care, vol. 13, n. 2, pp. 169-175, 2010. https://doi.org/10. $1007 /$ s12028-010-9405-7

[3] S. Delorme, D. Laroche, R. DiRaddo y R. F. Del Maestro, "NeuroTouch: a physics- based virtual simulator for cranial microneurosurgery training", Neurosurgery, vol. $71, \mathrm{n}^{\circ} .1$ suppl operative, pp. 32-42, 2012.

[4] M. Kersten-Oertel, S. S. J. Chen, S. Drouin, D. S. Sinclair y D. L. Collins, "Augmented reality visualization for guidance in neurovascular surgery", Stud. Health Technol Inform., vol. 173, pp. 225-229, 2012.

[5] Z. R. Yaniv, R. J. Webster, W. Fenz y J. Dirnberger, "Real-time surgery simulation of intracranial aneurysm clipping with patient-specific geometries and haptic feedback", Proceedings SPIE, vol. 9415, $\mathrm{n}^{\circ} .1,2015$.

[6] M. T. Lawton, "Seven Aneurysms: Tenets and Techniques for Clipping", Stuttgart: Thieme, 2011. https://doi.org/10.1055/b-002-66278

[7] O. R. Faha, "OSIRIX: An Open Source Platform for Advanced Multimodality Medical Imaging" 4th International Conference on Information \& Communications Technology, Information \& Communications Technology, Cairo, 2006.

[8] P. Cignoni, M. Callieri, M. Corsini, M. Dellepiane, F. Ganovelli, y G. Ranzuglia, "MeshLab: an Open-Source Mesh Processing Tool," Eurographics Italian Chapter Conference, Salerno, 2008.

[9] A. Updegrove, N. M. Wilson, J. Merkow, H. Lan, A. L. Marsden y S. C. Shadden, "SimVascular: An Open Source Pipeline for Cardiovascular Simulation", Annals of Biomedical Engineering, vol. 45, $\mathrm{n}^{\circ}$. 3, pp. 525-541, 2017. https://doi.org/10.1007/ s10439-016-1762-8

[10] F. Faure, "SOFA: A Multi-Model Framework for Interactive Physical Simulation", Soft Tissue Biomechanical Modeling for Computer Assisted Surgery, vol. 11, 283-321, 2012. https://doi.org/ $10.1007 / 8415 \_2012 \_125$

[11] M. Macklin, M. Müller, N. Chentanez y K. Tae-Yong, "Unified particle physics for real-time applications", ACM Transactions on Graphics, vol. 33, $\mathrm{n}^{\circ}$. 4. pp. 1-12, 2014. https://doi.org/10. $1145 / 2601097.2601152$ 
[12] J. Bender, M. Müller, M. A. Otaduy, M. Teschner, y M. Macklin, "A Survey on Position-Based Simulation Methods in Computer Graphics", Computer Graphics Forum, vol. 33, n ${ }^{\circ}$ 6. pp. 228-251, 2014. https://doi.org/10.1111/cgf.12346

[13] M. Bro-Nielsen y S. Cotin, "Real-time Volumetric Deformable Models for Surgery Simulation using Finite Elements and Condensation", Computer Graphics Forum, vol. 15, n ${ }^{\circ}$ 3, p. 57, 1996. https: //doi.org/10.1111/1467-8659.1530057

[14] M. Bro-Nielsen, "Finite element modeling in surgery simulation", Proceedings of the IEEE, vol. $86, \mathrm{n}^{\circ}$. 3, pp. 490-503, 1998. https://doi.org/10.1109/5. 662874

[15] J. Georgii y R. Westermann, "Corotated Finite Elements Made Fast and Stable", Workshop in Virtual Reality Interactions and Physical Simulation (VRIPHYS), Grenoble, 2008.

[16] M. Nesmea y F. Faurea, "Physically realistic interactive simulation for biological soft tissues", Recent Research Developments in Biomechanics, vol 2, pp.1-22, 2005. 